ARTICLE OPEN

Check for updates

\title{
Exosomal tau with seeding activity is released from Alzheimer's disease synapses, and seeding potential is associated with amyloid beta
}

Emily Miyoshi (iD ${ }^{1,11}$, Tina Bilousova ${ }^{1,2,3,11}$, Mikhail Melnik ${ }^{1,4}$, Danyl Fakhrutdinov ${ }^{1}$, Wayne W. Poon (iD ${ }^{5}$, Harry V. Vinters ${ }^{2,3,6}$, Carol A. Miller ${ }^{7,8}$, Maria Corrada ${ }^{5,9}$, Claudia Kawas ${ }^{5,9,10}$, Ryan Bohannan ${ }^{5}$, Chad Caraway ${ }^{5}$, Chris Elias ${ }^{2,3}$, Katherine N. Maina ${ }^{2,3}$, Jesus J. Campagna ${ }^{2,3}$, Varghese John ${ }^{2,3}$ and Karen Hoppens Gylys ${ }^{1,2,4}$

(c) The Author(s) 2021

Synaptic transfer of tau has long been hypothesized from the human pathology pattern and has been demonstrated in vitro and in vivo, but the precise mechanisms remain unclear. Extracellular vesicles such as exosomes have been suggested as a mechanism, but not all tau is exosomal. The present experiments use a novel flow cytometry assay to quantify depolarization of synaptosomes by $\mathrm{KCl}$ after loading with FM2-10, which induces a fluorescence reduction associated with synaptic vesicle release; the degree of reduction in cryopreserved human samples equaled that seen in fresh mouse synaptosomes. Depolarization induced the release of vesicles in the size range of exosomes, along with tetraspanin markers of extracellular vesicles. A number of tau peptides were released, including tau oligomers; released tau was primarily unphosphorylated and C-terminal truncated, with $A \beta$ release just above background. When exosomes were immunopurified from release supernatants, a prominent tau band showed a dark smeared appearance of SDS-stable oligomers along with the exosomal marker syntenin-1, and these exosomes induced aggregation in the HEK tau biosensor assay. However, the flow-through did not seed aggregation. Size exclusion chromatography of purified released exosomes shows faint signals from tau in the same fractions that show a CD63 band, an exosomal size signal, and seeding activity. Crude synaptosomes from control, tauopathy, and AD cases demonstrated lower seeding in tauopathy compared to $A D$ that is correlated with the measured $A \beta 42$ level. These results show that $A D$ synapses release exosomal tau that is $C$-terminal-truncated, oligomeric, and with seeding activity that is enhanced by $A \beta$. Taken together with previous findings, these results are consistent with a direct prion-like heterotypic seeding of tau by $A \beta$ within synaptic terminals, with subsequent loading of aggregated tau onto exosomes that are released and competent for tau seeding activity.

Laboratory Investigation (2021) 101:1605-1617; https://doi.org/10.1038/s41374-021-00644-z

\section{INTRODUCTION}

Of the two hallmark lesions of Alzheimer's disease (AD), the aggregated tau protein in neurofibrillary tangles correlates best with cognitive dysfunction. In AD, NFT pathology spreads in a stereotypic pattern, beginning in entorhinal cortex, moving to the hippocampus, and eventually to neocortex ${ }^{1}$. For this reason, transsynaptic spread of tau pathology between regions has long been hypothesized and more recently been shown convincingly in vitro ${ }^{2-4}$ and in vivo ${ }^{5}$. The importance of the synapse in transfer is highlighted by the result that synaptic contacts are required for exosome-mediated transmission of tau in vitro $^{6}$, and increased activity stimulates release and enhances tau pathology in vivo ${ }^{7}$. Along this line, work from our lab has shown that tau protein is abundant in control and $A D$ synapses, and is released by in vitro depolarization of AD synaptosomes ${ }^{8}$.
Exosomes are extracellular vesicles (EVs) of endocytic origin, usually defined by a size range $\sim 30-120 \mathrm{~nm}$, and by content of endosome-associated proteins ${ }^{9}$. Recent evidence suggests exosomes as a mechanism for prion-like spread of misfolded proteins in neurodegeneration ${ }^{10,11}$, and exosome-associated spread of tau pathology has been shown in the rTg4510 mouse and several other model systems $s^{6,12,13}$. However, released tau is found to be free-floating as well as localized to plasma-membrane-derived EVs, microvesicles, or ectosomes ${ }^{14}$, and the degree of exosomal localization varies with model system ${ }^{13,15}$. Similarly, results vary with respect to the degree of phosphorylation and truncation of released tau ${ }^{6,12,16,17}$. The size of the tau aggregate also affects propagation, with trimers being a minimal unit for seeding aggregation $^{18}$; in another study, soluble high molecular weight (HMW) p-tau peptides were rare, but were the key species for

\footnotetext{
${ }^{1}$ UCLA School of Nursing, UCLA School of Medicine, Los Angeles, CA, USA. ${ }^{2}$ Mary S. Easton Center for Alzheimer's Research at UCLA, UCLA School of Medicine, Los Angeles, CA USA. ${ }^{3}$ Department of Neurology, UCLA School of Medicine, Los Angeles, CA, USA. ${ }^{4}$ Neuroscience Interdepartmental Program, UCLA School of Medicine, Los Angeles, CA, USA. ${ }^{5}$ Institute for Memory Impairments and Neurological Disorders, UC Irvine, Irvine, CA, USA. ${ }^{6}$ Department of Pathology and Laboratory Medicine, UCLA School of Medicine, Los Angeles, CA, USA. ${ }^{7}$ Department of Pathology, Keck USC School of Medicine, Los Angeles, CA, USA. ${ }^{8}$ Department of Neurology, Program in Neuroscience, Keck USC School of Medicine, Los Angeles, CA, USA. ${ }^{9}$ Department of Neurology, UC Irvine, Irvine, CA, USA. ${ }^{10}$ Department of Neurobiology and Behavior, UC Irvine, Irvine, CA, USA. ${ }^{11}$ These authors contributed equally: Emily Miyoshi, Tina Bilousova. ${ }^{\circledR}$ email: kgylys@sonnet.ucla.edu
}

Received: 25 August 2020 Revised: 7 July 2021 Accepted: 9 July 2021

Published online: 30 August 2021 
Table 1. Case information.

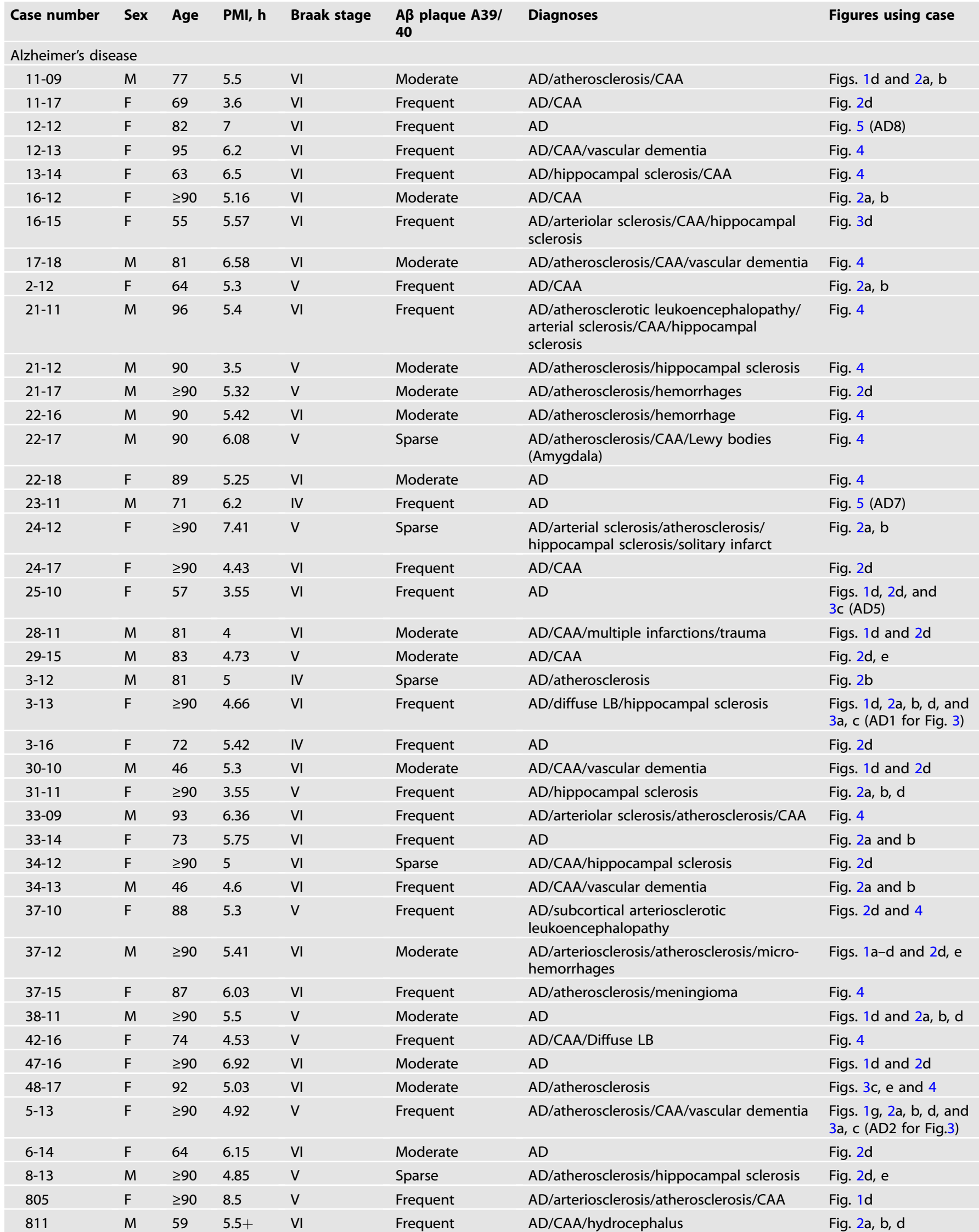


Table 1 continued

\begin{tabular}{|c|c|c|c|c|c|c|c|}
\hline Case number & Sex & Age & PMI, h & Braak stage & $\begin{array}{l}A \beta \text { plaque } A 39 / \\
40\end{array}$ & Diagnoses & Figures using case \\
\hline 869 & $\mathrm{~F}$ & 75 & 5 & $\mathrm{VI}$ & Moderate & $A D$ & Fig. $2 a, b$ \\
\hline $9-18$ & $\mathrm{~F}$ & 89 & 4.87 & V & Frequent & $\begin{array}{l}\mathrm{AD} / \text { ischemic leukoencephalopathy/ } \\
\text { atherosclerosis/vascular dementia }\end{array}$ & Fig. 4 \\
\hline 909 & M & 86 & $5.5+$ & V & Frequent & $\mathrm{AD} / \mathrm{CAA} /$ ependymitis & Fig. $2 d$ \\
\hline U1 & $\mathrm{F}$ & 96 & 5 & $\mathrm{VI}$ & Frequent & $\mathrm{AD} / \mathrm{CAA} /$ cerebrovascular disease & Fig. $2 d$ \\
\hline U2 & M & 82 & 5 & $\mathrm{~V}-\mathrm{VI}$ & Moderate & $\mathrm{AD} / \mathrm{CAA} /$ cerebrovascular disease & Figs. $1 d$ and $2 a, b$ \\
\hline $7-11$ & $\mathrm{~F}$ & $\geq 90$ & 4.25 & III & Moderate & CIND/atherosclerosis & Fig. 5 (AD6) \\
\hline \multicolumn{8}{|l|}{ Tauopathy } \\
\hline U3 & M & 75 & 8 & IV & None & $\begin{array}{l}\text { NFT-predominant AD/arteriosclerosis/ } \\
\text { atherosclerosis }\end{array}$ & Fig. 5 (T1) \\
\hline \multicolumn{8}{|l|}{ Controls } \\
\hline $1-13$ & M & $\geq 90$ & 6 & 1 & None & Atherosclerosis/solitary infarct & Fig. 5 (N1) \\
\hline 830 & $\mathrm{~F}$ & 89 & 4.25 & II & None & $\mathrm{AD} /$ vascular dementia/atrophy & Fig. 3a (Con) \\
\hline 907 & M & 84 & 5 & $N / A$ & None & Normal & Fig. 5 (N2) \\
\hline
\end{tabular}

Plaques number in the area (based on Bielschowsky stain and IHC): none $=0$; sparse $=1-5$; moderate $=6-20$; frequent $=$ more than 20 .

CIND cognitive impairment, no dementia, CAA cerebral amyloid angiopathy.

propagation $^{19,20}$. Like amyloid $\beta(A \beta)$, tau protein forms oligomeric intermediates that mediate downstream toxicity; we and others have shown tau oligomers localized to synapses ${ }^{21-23}$. A $\beta$ is also found in exosomes, and has been shown to propagate by exosomes $^{24}$. Blockade of exosomal pathways has been shown to reduce plaque pathology $y^{25}$, and suggested as a possible therapeutic approach for reduction of tau propagation ${ }^{26}$. Because tau, $p$-tau, and $A \beta$ are elevated in brain-derived exosomes from plasma, exosomes have also been proposed as blood-based biomarkers for $\mathrm{AD}^{27}$.

$A \beta$ immunotherapy has generally not shown efficacy in humans, but both active and passive tau immunotherapy has shown encouraging results in at least 13 studies in animal models ${ }^{16,28,29}$; tau immunotherapy has been shown to reduce amyloid as well as tau pathology ${ }^{30}$. However, a primary challenge for tau-based immunotherapy approaches is the lack of understanding of the precise form of the tau peptide(s) that is neurotoxic ${ }^{28}$. Based on our previous work using cryopreserved postmortem $A D$ synaptosomes and the lack of consensus in the literature, we have developed an assay to quantify depolarization of in vitro synaptosomes, and demonstrated depolarizationinduced release of tau and exosome-like EVs from AD synaptosomes. Released tau peptides from AD synapses are largely Cterminal-truncated, unphosphorylated, and oligomeric. Exosomal tau and tau oligomers are increased in $A D$ compared to control exosomes, and exosomes released from $A D$ synapses demonstrate elevated seeding activity that is enhanced by $A \beta$ in a FRET-based biosensor assay.

\section{MATERIALS AND METHODS}

\section{Human brain specimens}

The primary goal of the present experiments was to characterize tau and exosomes released from $A D$ synaptosomes; due to the requirement for large sample volume, only a few control brains were used for key experiments. Pilot experiments showed a higher degree of depolarization for samples with relatively short postmortem interval (PMI) $(<6 \mathrm{~h})$; therefore samples were chosen based on PMI and relatively similar levels of $A D$ pathology. Frontal and parietal cortex (Brodmann areas A7, A9, A39, A40) samples were obtained at autopsy from $A D$ research centers at UCLA, $\mathrm{UCl}$, and USC (Table 1$)$. Immediately on receipt, samples $(\sim 0.3-5 \mathrm{~g})$ were minced in a $0.32 \mathrm{M}$ sucrose solution with protease inhibitors for cryopreservation of synaptic structure and membranes ${ }^{31}$ (2 mM EDTA, 2 mM EGTA, $0.2 \mathrm{mM}$ PMSF, $1 \mathrm{mM}$ Na pyrophosphate, $5 \mathrm{mM} \mathrm{NaF}$, $10 \mathrm{mM}$ Tris), then stored at $-80^{\circ} \mathrm{C}$ until homogenization. The P-2 (crude synaptosome; synaptosome-enriched fraction) was prepared as previously described ${ }^{32}$; briefly, tissue was homogenized in ice cold buffer $(0.32 \mathrm{M}$ sucrose, $10 \mathrm{mM}$ TRIS pH 7.5, plus protease inhibitors: pepstatin $(4 \mu \mathrm{g} / \mathrm{ml})$, aprotinin $(5 \mu \mathrm{g} /$ $\mathrm{ml})$, trypsin inhibitor $(20 \mu \mathrm{g} / \mathrm{ml})$, EDTA $(2 \mathrm{mM})$, EGTA $(2 \mathrm{mM})$, PMSF $(0.2$ $\mathrm{mM})$, Leu-peptin $(4 \mu \mathrm{g} / \mathrm{ml})$ ). The homogenate was first centrifuged at 1000 $g$ for $10 \mathrm{~min}$; the resulting supernatant was centrifuged at $10,000 \mathrm{~g}$ for 20 min to obtain the crude synaptosomal pellet. Aliquots of $\mathrm{P}-2$ are routinely cryopreserved in $0.32 \mathrm{M}$ sucrose and banked at $-80^{\circ} \mathrm{C}$ until the day of the experiment ${ }^{31}$, at which time they were defrosted at $37^{\circ} \mathrm{C}$.

\section{Mouse brain specimens}

WT mice expressing human apoE (E3 and E4) from a previous study were euthanized, and cortices $(\sim 0.1-0.15 \mathrm{~g})$ were processed into P-2 immediately. In order to examine differences between fresh and frozen samples, mouse P-2 samples were not cryopreserved and were immediately used for our depolarization assay.

\section{Depolarization assay}

For preparation of buffers used in our depolarization assay, ASTM type 1 water (LabChem) was used. After defrosting, human P-2 samples were centrifuged at $10,000 \mathrm{~g}$ for $10 \mathrm{~min}$ at $4{ }^{\circ} \mathrm{C}$ to remove sucrose; typical experiments used two $0.3 \mathrm{ml}$ aliquots ( $25.8 \mathrm{mg}$ tissue). Samples were then resuspended in Normal Krebs buffer $(160 \mathrm{mM} \mathrm{NaCl}, 5.5 \mathrm{mM} \mathrm{KCl}, 10 \mathrm{mM}$ HEPES, $10 \mathrm{mM}$ glucose, $10 \mathrm{mM}$ pyruvate, $1.2 \mathrm{mM} \mathrm{MgCl} 2,1.5 \mathrm{mM} \mathrm{CaCl}_{2}$ ) and incubated at $37^{\circ} \mathrm{C}$ for $5 \mathrm{~min}$. Samples were divided in order to measure 
Table 2. Reagents.

\begin{tabular}{|c|c|c|c|c|}
\hline Antibody name & Antigen/epitope & Supplier & Host & Reactivity \\
\hline FM2-10 (dye) & $\begin{array}{l}\text { Associates with cellular } \\
\text { membranes }\end{array}$ & Invitrogen (Waltham, MA) & N/A & N/A \\
\hline Calcein AM (dye) & $\begin{array}{l}\text { Intact cells/synaptosomes } \\
\text { (viability dye) }\end{array}$ & Biolegend (San Diego, CA) & N/A & N/A \\
\hline $\begin{array}{l}\text { Exosme-anti-CD63 for } \\
\text { western (TS63) }\end{array}$ & CD63 & Invitrogen (Waltham, MA) & Mouse & Human \\
\hline $\begin{array}{l}\text { Exosome-anti-CD81 for } \\
\text { Western (M38) }\end{array}$ & CD81 & Invitrogen (Waltham, MA) & Mouse & Human \\
\hline Anti-HSP70 antibody & HSP70 & $\begin{array}{l}\text { SBI System Biosciences (Palo } \\
\text { Alto, CA) }\end{array}$ & Rabbit & Human \\
\hline Tau monoclonal antibody (HT7) & $\begin{array}{l}\text { Tau (including PHF and non- } \\
\text { PHF tau) }\end{array}$ & Invitrogen (Waltham, MA) & Mouse & Human, Bovine \\
\hline Anti-Tau(22), oligomeric antibody & Oligomeric Tau & Millipore (Burlington, MA) & Rabbit & Human \\
\hline $\begin{array}{l}\text { Recombinant anti-syntenin } \\
\text { antibody }\end{array}$ & Syntenin-1 & Abcam (Cambridge, UK) & Rabbit & Human \\
\hline
\end{tabular}

depolarization with FM2-10; simultaneously, release fractions were collected for biochemical analysis and exosome purification. Based on a previous protocol for $\mathrm{FM} 2-10^{33}$, P-2 aliquots $(100 \mu \mathrm{l})$ were incubated with FM2-10 $(25 \mu \mathrm{M})$ for a minute before a $2 \mathrm{~min} 30 \mathrm{mM} \mathrm{KCl}$ stimulation at $37^{\circ}$ C. Excess dye was then washed out with $1 \mathrm{mg} / \mathrm{ml} \mathrm{BSA}$, and the samples were centrifuged at $10,000 \mathrm{~g}$ for $10 \mathrm{~min}$ at room temperature. A second wash was performed with Normal Krebs before resuspending and dividing each sample between two flow cytometry tubes. Fifty $\mathrm{mM}$ final $\mathrm{KCl}$ was added to one tube, and both tubes were read on BD FACSCalibur (BectonDickinson, San Jose, CA) at 5, 10, and 20 min timepoints. FM2-10 and calcein AM fluorescence was plotted against forward scatter, which is proportional to size. An analysis gate was drawn on forward scatter (FSC), based on size standards $(0.75-1.5 \mu \mathrm{m})$, to ensure that fluorescence was quantified only in particles within the size range of synaptosomes ${ }^{34,35}$. Flow cytometry data were analyzed using FCS Express version 5 software (DeNovo Software California, USA).

The remaining P-2 samples for biochemistry/exosome isolation were incubated at $37^{\circ} \mathrm{C}$ for $3 \mathrm{~min}$ and centrifuged. Samples were resuspended in Normal Krebs and divided between two tubes. Equal volumes of Normal Krebs and $50 \mathrm{mM}$ final $\mathrm{KCl}$ were added to corresponding tubes and incubated for $5 \mathrm{~min}$ at $37^{\circ} \mathrm{C}$ before immediate addition of protease and phosphatase inhibitors (Fisher, Waltham, MA) followed by centrifugation at $10,000 \times g$ at $4^{\circ} \mathrm{C}$. Supernatants were collected and either immediately use for fractionation/concentration with Vivaspin 500 centrifugal concentrators (Sartorius, Gottingen, Germany) and/or exosome isolation or stored at $-80^{\circ} \mathrm{C}$. Total protein concentration was determined with the Pierce BCA assay.

\section{Western and dot blotting}

Collected supernatants were separated by gel electrophoresis on $10-20 \%$ Tris-Glycine gradient gels with $4 x$ Tris-Glycine sample buffer. After transferring to Immobilon-P membrane, membranes were blocked with $5 \%$ BSA for $1 \mathrm{~h}$, and primary antibodies were incubated overnight at $4{ }^{\circ} \mathrm{C}$. Antibodies used are listed in Table 2. For dot blotting, samples were applied to a nitrocellulose membrane using the Bio-Dot microfiltration apparatus. The membrane was blocked with $5 \%$ milk for $1 \mathrm{~h}$, and the primary antibody incubated overnight at $4{ }^{\circ} \mathrm{C}$. After secondary antibody incubation, immunolabeled proteins were visualized and quantified by SuperSignal West Femto maximum sensitivity substrate (Thermo Scientific, Rockford, IL) on a UVP BioSpectrum 600 imaging system using VisionWorks Version 6.6A software (Upland, CA). Human recombinant tau441 protein preformed fibrils (PFF) used in control experiments were purchased from StressMarq Biosciences (SPR-475B, Victoria, Canada); for each experiment the aliquot was divided in two equal parts and $1 / 2$ was sonicated for 10 min in water bath sonicator therefore the added tau contained a mixture of different size tau fibrils and oligomers.

\section{Transmission electron microscopy (TEM)}

Collected supernatants from the depolarization assay were concentrated using Vivaspin 500 centrifugal concentrators (Sartorius, 100,000 MWCO). The concentrate was fixed on a copper mesh in glutaraldehyde/paraformaldehyde solution followed by staining with $2 \%$ uranyl acetate solution and imaged on JEOL $100 C X$ electron microscope at $\times 29,000$ magnification.

\section{Exosome isolation: immunoprecipitation}

Antibodies for three general exosomal surface markers: tetraspanins CD63 (TS63), CD9 (TS9), and CD81 (M38) from Invitrogen were first coupled to superparamagnetic beads (Dynabeads MP-270 Epoxy; ThemoFisher) using a dynabeads antibody coupling kit (ThermoFisher), according to the manufacturer's instructions. To ensure maximal exosomal yield during immunoprecipitation (IP), anti-CD63, anti-CD9, and anti-CD81, beads were added simultaneously to the samples in final concentration $0.1 \mathrm{mg} / \mathrm{ml}$ for each type of beads (pan-specific isolation) followed by overnight rotation at $4{ }^{\circ} \mathrm{C}$. After four washes with PBS, exosomes were lysed by $10 \mathrm{~min}$ boiling in $50 \mu \mathrm{l}$ of $1 x$ Novex Tris-Glycine SDS Sample buffer with DTT $(200 \mu \mathrm{M})$. Beads were separated by magnet and IP samples were run in 10-20\% SDS PAGE (Invitrogen) followed by immunoblotting analysis with syntenin-1 $(\mathrm{H}-$ 48, Santa Cruz Biotech) and tau (HT7, Invitrogen) antibodies. Standard human plasma exosomes were purchased (Galen Laboratory Supplies, Middletown, CT), and loaded as a positive control.

\section{Exosome isolation: ultracentrifugation}

The supernatant was spun at $2000 \times g$ for $10 \mathrm{~min}$, then the supernatant was collected and spun at $10,000 \times g$ for $30 \mathrm{~min}$ at $4^{\circ} \mathrm{C}$. Supernatant from the last centrifugation was applied to a triple sucrose cushion gradient for ultracentrifugation $\left(80,000 \times g\right.$ for $3 \mathrm{~h}$ at $\left.4^{\circ} \mathrm{C}\right)$. Fractions $1-3$ were collected as described ${ }^{36}$, and diluted for a final spin $\left(100,000 \times g\right.$ for $1 \mathrm{~h}$ at $\left.4^{\circ} \mathrm{C}\right)$ to pellet vesicles, then suspended in $25 \mathrm{mM}$ trehalose/PBS to prevent aggregation and cryodamage, then slow frozen at $-80^{\circ} \mathrm{C}$. EV size was determined using tunable resisting pulse sensing analysis using a qNano Gold instrument using nanopore NP100 (Izon Science, Medford, MA).

\section{Exosome isolation: size exclusion chromatography (SEC)}

Supernatants collected after synaptosome depolarization (150 or $300 \mu \mathrm{l}$ of supernatants from P-2 weight equal $0.04 \mathrm{~g}$ or $0.12 \mathrm{~g}$, respectively) were loaded 


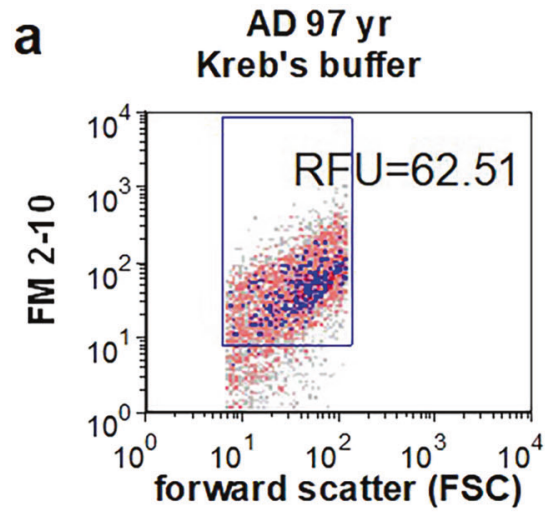

b $\quad \begin{array}{rl}\mathrm{AD} & 97 \mathrm{yr} \\ \mathrm{KCl} & 50 \mathrm{mM}\end{array}$
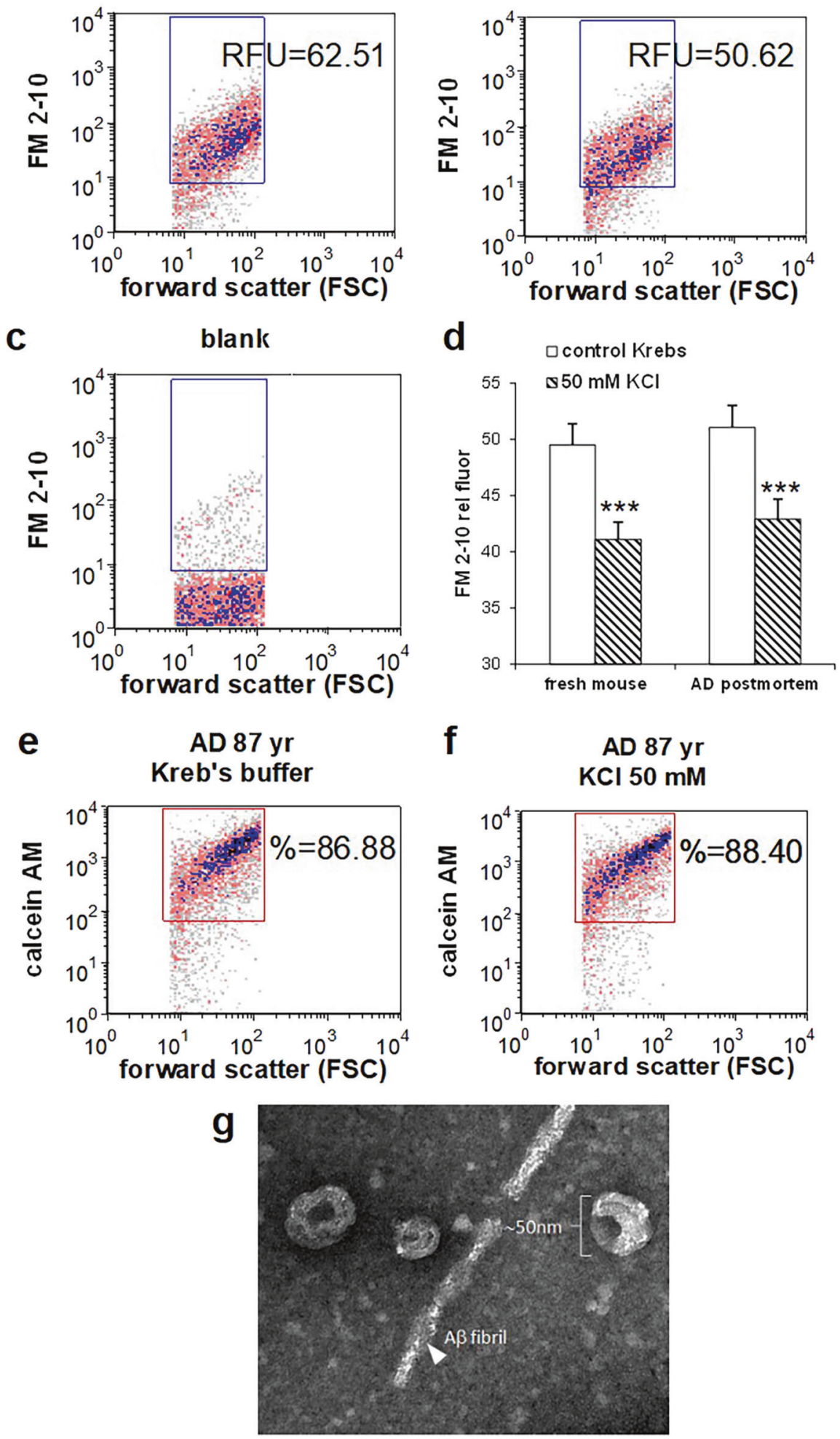

Fig. 1 Flow cytometry assay for in vitro quantification of depolarization. AD cortical synaptosomes were incubated with FM2-10 (25 $\mu M$ ) prior to incubation with $\mathrm{KCl}(30 \mathrm{mM})$ and flow cytometry analysis. Representative density plots illustrate FM2-10 labeling in baseline (a) and depolarized samples (b); reduction in fluorescence corresponds to exocytic activity. Forward scatter (FSC) is proportional to particle size; rectangular analysis gate is drawn on size standard to include particles from $\sim 0.5$ to $1.5 \mu \mathrm{m}$, data collected from 5000 events. c Background labeling in unstained blank. d Aggregate data from human AD cortex (A7 or A9; $n=13)$ and fresh mouse cortex $(n=12 ; p<0.0001)$. e Representative density plots illustrate viability dye calcein $A M$, showing integrity of baseline (e) and depolarized (f) P-2 samples. $\mathbf{g}$ AD release supernatant was concentrated as described in "Materials and methods" for transmission electron microscopy. 
to prewashed qEVsingle/35 nm SEC columns (Izon Science, Medford, MA) and fraction were collected according to manufacturer's instructions. Briefly, after void volume $(1 \mathrm{ml})$ was discard, five fractions of $200 \mu \mathrm{l}$ each (F5, F6, F7, F8, and F9) were collected. Halt's protease and phosphatase inhibitor cocktail (ThermoFisher, Waltham, MA) were added and the fractions were aliquoted and kept at $-80^{\circ} \mathrm{C}$. For control experiments one aliquot of tau PFF (StressMarq Biosciences, Victoria, Canada), contained $10 \mu \mathrm{g}$ of tau PFF in $10 \mu \mathrm{l}$ PBS, was sonicated for $10 \mathrm{~min}$ and then mixed with an aliquot of tau PFF (which was not sonicated) and $20 \mu \mathrm{g}$ of standard human plasma exosomes (Galen Laboratory Supplies, Middletown, CT), volume of mixture was adjusted to $150 \mu \mathrm{l}$ and loaded to qEVsingle/35 nm SEC columns and fractions were collected as described above. In parallel, the same amount of tau PFF $(20 \mu \mathrm{g})$ was processed in an exact same way but was not mixed with exosomes before applying to a qEV column.

\section{Tau biosensor cell assay}

We used the HEK293T Tau RD P301S FRET biosensor (tau biosensor) assay according to the previously described protocol ${ }^{37}$. Briefly, samples were sonicated for $10 \mathrm{~min}$ and transduced to tau biosensors using Lipofectamine 2000 (ThermoFisher Scientific, Walthman, MA). Cells were harvested at the $60 \mathrm{~h}$ timepoint and fixed using $2 \%$ solution of paraformaldehyde. An Attune NxT Flow Cytometer (Invitrogen) equipped with an autosampler and FRET-compatible laser lines and filter sets was used for FRET signal detection. The FRET (CFP/YFP) signal was excited by a $405 \mathrm{~nm}$ laser for CFP excitation and detected in the YFP image detection channel. Flow cytometry data were analyzed using FCS Express version 5 software (DeNovo Software California, USA). Integrated FRET density was calculated as a product of percentage FRET positive cells and median of fluorescent intensity of the FRET positive cells as previously established ${ }^{37}$.

\section{Statistical analysis}

Data are presented as mean \pm SEM. All comparisons between control and depolarized samples used a Student's $t$ test for paired samples unless otherwise noted. One-way analysis of variance with Tukey HSD post hoc test was used for experiments with three or more comparison groups.

\section{RESULTS \\ The styryl dye FM2-10 quantifies synaptosome depolarization in vitro}

Synaptosomes are resealed nerved terminals prepared from fresh brain tissue that contain mitochondria and maintain membrane potential, along with a full complement of receptor and transporter functions ${ }^{38}$. Functional endpoints such as LTP and glucose transport can also be measured in postmortem human synaptosomes, if prepared from tissue that is cryopreserved by mincing and slow freezing in isotonic sucrose $\mathrm{s}^{39-43}$. In order to verify and quantify depolarization in postmortem $A D$ synaptosomes, we used FM2-10, a styryl dye with a lipophilic tail that becomes fluorescent on insertion into membranes ${ }^{44,45}$. Based on a previous exocytosis assay ${ }^{33}$, we developed a flow cytometry assay to measure synaptosome depolarization in vitro. P-2 samples (crude synaptosomes, synaptosome-enriched fraction) prepared from cryopreserved AD cortex were first loaded with FM2-10 dye, then depolarization was detected as a reduction in FM2-10 fluorescence reflecting vesicle release after depolarization. Dot plots present FM2-10 fluorescence vs. FSC, which is the light scattering parameter proportional to size; the blue rectangular gate is drawn based on size standards to include only particles in the size range of synaptosomes ${ }^{34}$. The assay is illustrated by representative cortical $A D$ samples showing baseline fluorescence in control Kreb's buffer (62.51 RFU; Fig. 1a), which was reduced to 50.62 by the addition of $30 \mathrm{mM} \mathrm{KCl}$ to the buffer for $5-20 \mathrm{~min}$ (Fig. 1b). Figure 1c illustrates typical background labeling. The mean maximum fluorescence was reduced from 51.1 to 42.9 RFU $(\sim 16 \% ; p<0.0001$; Fig. 1d). Because the experimental goal was clarification of synaptically released exosomes in $A D$, late stage $A D$ cases were used for initial experiments (Braak stage V-Vl; Table 1) with a mean PMI $5.3 \mathrm{~h}( \pm 0.4)$. To serve as non-AD controls, and to determine if the cryopreservation step for human samples affects subsequent depolarization, we next measured depolarization of mouse synaptosomes prepared immediately after sacrifice. FM2-10 fluorescence levels for baseline and depolarized mouse cortical synaptosomes were very similar to results with human samples, with a mean maximum baseline of 49.52 RFU that was reduced to 41.12 by depolarization ( $p<0.0001$; Fig. $1 \mathrm{~d}$ ). This result indicates that sucrose cryopreservation of minced tissue at the time of autopsy preserves membrane potential and exocytotic function to a level comparable to that measured in fresh synaptosomes.

To ensure that in vitro incubation with $30 \mathrm{mM} \mathrm{KCl}$ does not compromise synaptosomal membrane integrity and function, we next tested integrity of depolarized synaptosomes by labeling synaptosomes with the live cell marker calcein AM. Calcein AM is a lipophilic dye that enters cells readily and is converted to a polar fluorescent product by esterases; the dye is retained by synaptosomes with an intact membrane ${ }^{46}$. As shown in representative samples (Fig. 1e, f), calcein AM brightly labels $~ 90 \%$ of size-gated synaptosomes, and the positive fraction is not reduced by depolarization for $20 \mathrm{~min}\left(37^{\circ} \mathrm{C} ; 86.53 \pm 0.58 \%\right.$ pos vs. $87.15 \pm$ $1.3 \%$ pos). These results demonstrate the integrity of in vitro synaptosomes in our flow cytometry assay, and precisely quantify the degree of synaptosomal depolarization.

\section{Depolarization induces release of EVs and tau from cortical synaptosomes}

Much evidence supports EV-mediated spread of tau pathology, and the tetraspanin protein family in particular is not only highly enriched in EVs, but shown to play a role in biogenesis, assembly, and recruitment of cargos to $\mathrm{EVs}^{47}$. Therefore, we examined synaptosome release supernatants from AD cortex for exosomes and for tetraspanin markers of EVs. Electron microscopy confirmed that EVs released from depolarized AD cortical synaptosomes demonstrate the morphology and relative size of exosomes $\left(30-120 \mathrm{~nm}^{9}\right.$; Fig. 1g). As shown in Fig. 2a, b, the tetraspanin markers for EVs, CD63, CD9, and CD81 are all increased following depolarization ( $p<0.01$; Student's paired $t$ test). The chaperone HSP70, sometimes used as an exosome marker, showed the same trend but was not significantly elevated by depolarization.

Tau is frequent exosome cargo, and a number of papers demonstrate the release of exosomal and free-floating tau in in vitro and in vivo tauopathy models $s^{6,12,13}$; therefore we next examined the nature of tau peptides released from $A D$ cortical synaptosomes. Consistent with previous results, the mid-region tau antibody HT7 labels most tau peptides and showed a relatively robust release of tau from synaptosomes (Fig. $2 c, d ; p<0.01$ ). The p-tau antibody pS422 shows that some released tau is phosphorylated, and also reveals a low level of phosphorylated tau oligomers the approximate size of a tau trimer (p-tau olig; Fig. 2c, d). However, multiple attempts to detect other p-tau epitopes (acetylated tau, caspase-cleaved tau, PHF1, p396, AT100) were not successful.

\section{Most tau released from AD synapses is C-terminal-truncated} The end-specific antibody tau 12 labels C-terminal-truncated tau (i.e., intact $\mathrm{N}$-terminus), and tau46 labels $\mathrm{N}$-terminal-truncated tau (i.e., intact C-terminus); release of both peptides was increased by depolarization (Fig. $2 \mathrm{c}, \mathrm{d} ; p<0.01$ ). Dot blots using the tau oligomer antibody $\mathrm{T} 22$ shows that tau oligomers are released from synaptosomes (Fig. 2c), confirming oligomeric bands observed in some samples with HT7 and tau46 (not shown); however, the depolarization-induced release trend is not significant. The aggregate data for EV markers (Fig. 2b, c) and for tau peptides (Fig. 2c, d) indicate that significant release of exosomes and tau occurs in resting synaptosomes, i.e., the increase induced by depolarization is fairly modest.

$A \beta$ has also been reported as an exosome cargo ${ }^{24,48}$, therefore multiple experiments measured $A \beta$ in $A D$ release supernatants. Several Western blots showed faint $A \beta$ bands on long exposure 

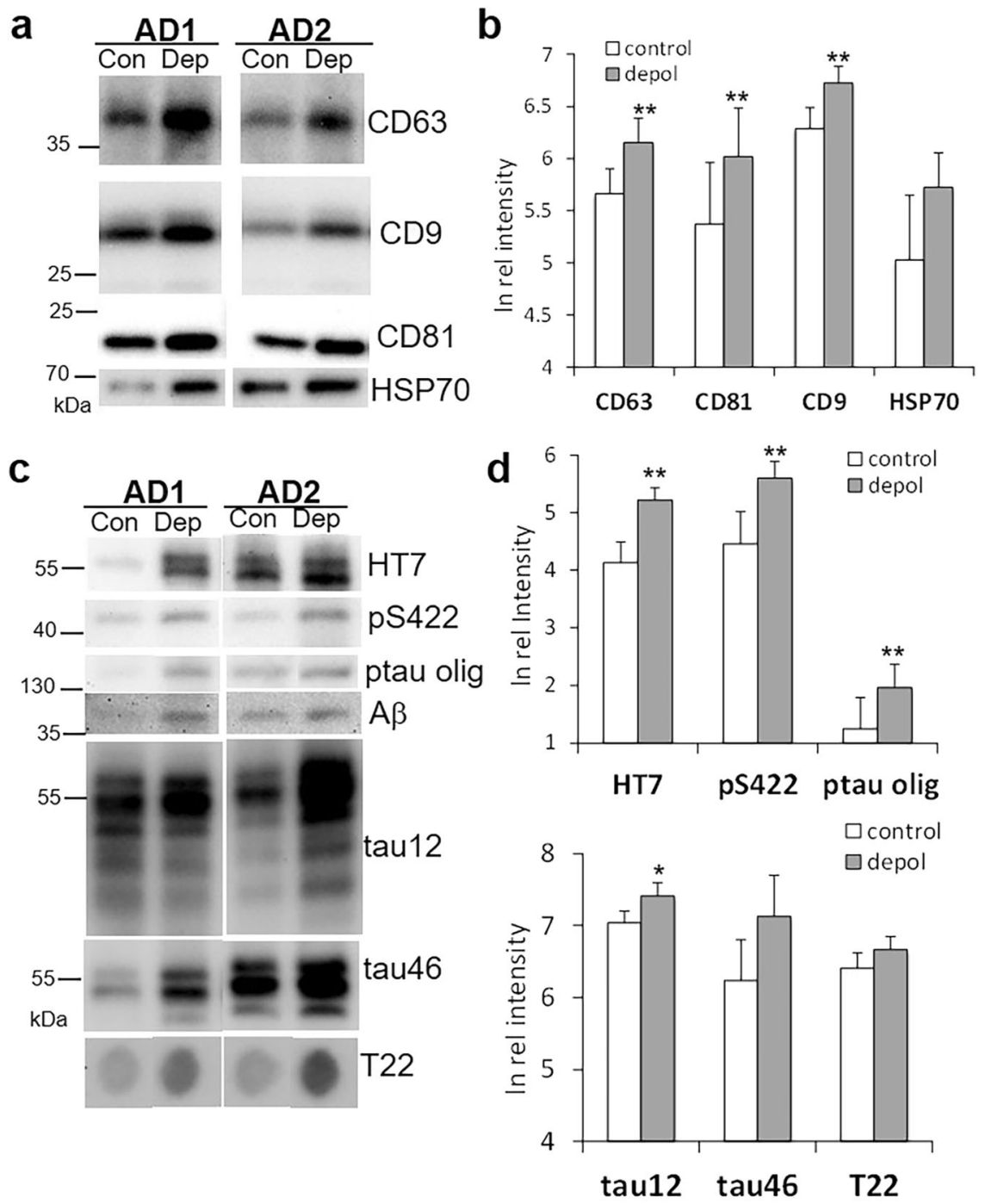

e

Tau12

Tau46

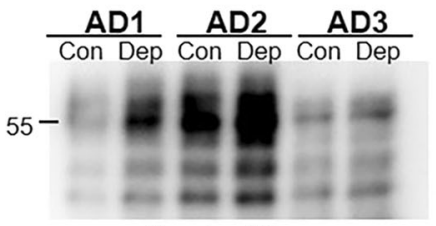

$5 \min 15 \mathrm{sec}$

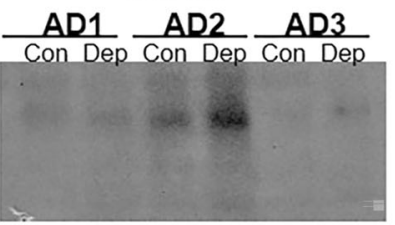

$20 \mathrm{~min} 15 \mathrm{sec}$

Fig. 2 Western SDS PAGE analysis of synaptosome release supernatants. a Representative immunoblots demonstrate labeling for markers of extracellular vesicles: levels of the tetraspanins CD63, CD9, CD81, and heat shock protein 70 (HSP70) are compared in control (con) and depolarized (dep) samples. b Aggregate data for (a); $n=7, p<0.01$. c Representative immunoblots for tau peptides (see Table 1 for antibodies); the $\mathrm{p}$-tau oligomer was immunolabeled with PS422. For T22 image is a dot blot. d Aggregate data for $(\mathbf{c}) ; n=6-13,{ }^{*} p<0.05,{ }^{* *} p<$ 0.01. e Immunoblots with tau12 (detects C-terminal truncated; intact N-terminus), and tau46 (detects N-terminal truncated; intact C-terminus) that include exposure times below blot, showing faint tau46 despite a fourfold longer exposure time. Blots shown are representative of five separate experiments with 3-7 cases/blot for each of the two antibodies.

(Fig. 2c); in multiple experiments, the band was observed $\sim 38 \mathrm{kDa}$, which is approximately the size of an apoE/A $\beta$ heterodimer, or possibly an $A \beta$ oligomer the size of 8-mer, both previously observed $^{49}$. However, $A \beta$ release was not enhanced by depolarization, and in AlphaLISA experiments, the signal was just above background, confirming the very low level of released $A \beta$ (not shown). As noted above, both $\mathrm{N}$-terminal and C-terminal tau were released from $A D$ synaptosomes; however, comparison of blots together with exposure times (Fig. 2e) shows a very faint full-length signal from tau with an intact C-terminus, compared to that with an intact $\mathrm{N}$-terminus, indicating that most tau released from $A D$ synaptosomes is C-terminal truncated. This result is consistent with our previous observation that synaptic tau lacks a C-terminus; in synaptosomes, those peptides with a C-terminal end are also generally full length $\left(\sim 55 \mathrm{kDa}^{50}\right)$.

\section{Tau released from cortical synapses is exosomal}

Because tau released from synaptosomes can be free-floating and/or exosomal, we next verified exosomal localization of tau. Starting with larger P-2 samples ( $200 \mathrm{mg}$ compared to $26 \mathrm{mg}$ ) from three $A D$ 
a

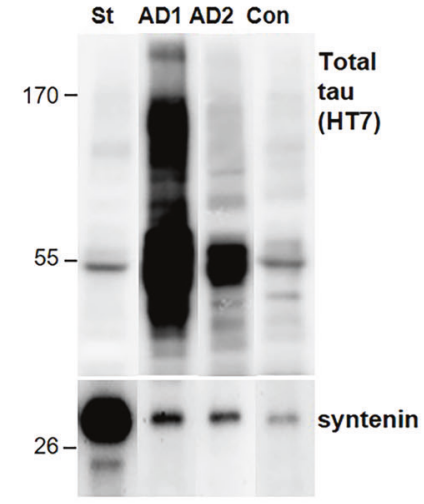

C

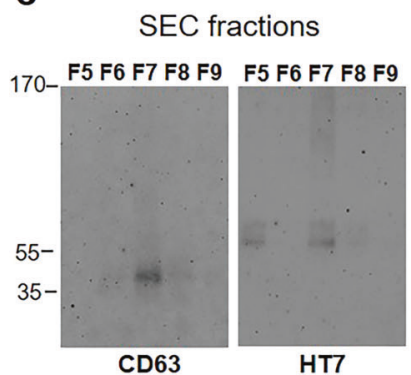

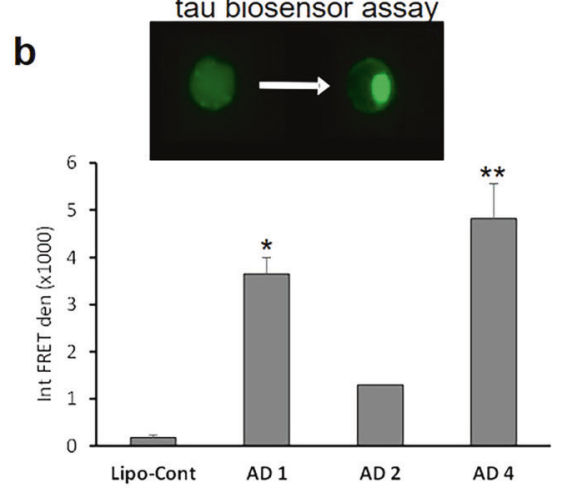

d

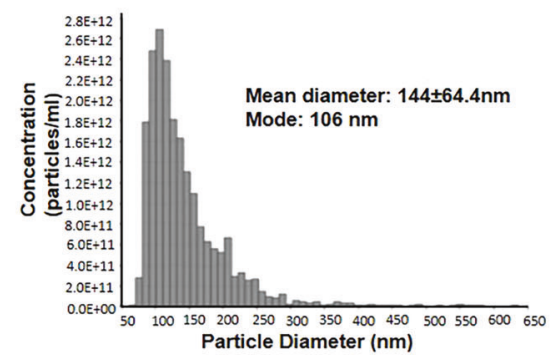

f SEC fractions (standard Exo and tau PFF)

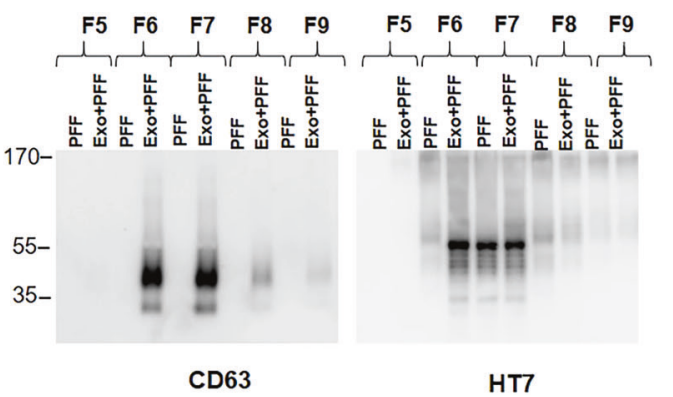

Fig. 3 AD cortical synapses release exosomal tau with seeding activity. a Large ( $200 \mathrm{mg})$ P-2 samples from two AD and one control (Con) cases were depolarized and exosomes from each case were purified by simultaneous immunoprecipitation (IP) with antibodies to CD63, CD9, and CD81 (pan exosome isolation). IP samples were immunolabeled with the HT7 antibody against tau and the exosome marker syntenin; the left lane control $(\mathrm{St})$ shows labeling with commercial standard human plasma exosomes. b To determine seeding activity, release supernatants from three P-2 samples were concentrated and loaded to HEK293T Tau RD P301S FRET biosensor cells (tau biosensor). Aggregate data (mean \pm SEM) are shown for the three AD cases along with lipofectamine control (lipo-cont), all in duplicate; ${ }^{*} p<0.05,{ }^{* *} p<0.01$, Students $t$ test for independent samples. c Representative Western SDS PAGE analysis of SEC fractions shows EV/exosome signal with antibody to tetraspanin CD63 and the total tau antibody HT7. d Representative tunable resistive pulse sensing (TRPS) analysis shows the size of particles in F7 fraction consistent with exosomes. e HEK293 tau biosensor assay for SEC fractions; integrated FRET density, Int FRET den, Cont is lipofectamine control, P-2 is crude synaptosome positive control. Error bars represent mean \pm SEM, $p<0.05$. f Western SDS PAGE of SEC fractions with added standard exosomes (Exo) plus commercial tau fibrils (PFF) alternating with added PFF alone.

cases, samples were depolarized as described above and supernatants were collected. Exosomes were purified from each release supernatant using immunoprecipitation with antibodies to the exosome-associated tetraspanins CD63, CD9, and CD81. Beads were separated by magnet and IP samples were immunoblotted with the HT7 antibody against tau. Commercial standard exosomes from healthy adult plasma were included as a positive control. The Western blot showed a strong band for full-length tau $(\sim 55 \mathrm{kDa})$ in two of the three cases (Fig. 3a), with a dark smear of fragments and oligomers in one case (AD1). As noted in Table 1, Case AD1 (3-13) showed advanced AD with diffuse Lewy bodies. Case AD2 (5-13), with strong $\sim 55 \mathrm{kDa}$ bands, was also an advanced AD case, with vascular dementia characteristics noted. The control case (Con; 830), with very little exosomal tau (Fig. 3a), showed little tau pathology (Braak II), no beta-amyloid pathology in parietal cortex, and severe atherosclerosis. This case was included as a control due to the relatively minimal neuropathologic change and the need for a large tissue volume. It is interesting that the standard plasma exosomes (lane 1, St), also have a small tau band at $\sim 55 \mathrm{kDa}$. The blot was reprobed for syntenin-1, a marker of syndecan-syntenin-alixdependent pathway of exosome biogenesis ${ }^{51}$, and suggested as a specific marker for small EVs representing "bona fide exosomes"9. The density of the syntenin-1 band from synaptic release is very small compared to that from healthy plasma control standard exosomes (lane St, left); together, these results clearly verify exosomal localization of tau peptides released from human $A D$ synapses. 

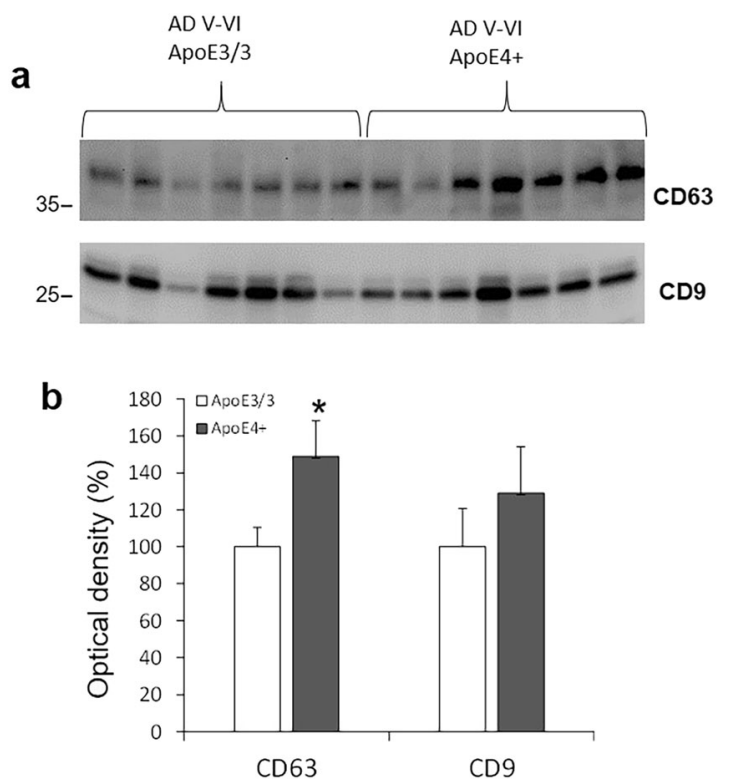

Fig. 4 Synaptically released exosomes are increased in cortex of APOE4 compared to APOE3. a Exosomes from release supernatants were purified and separated by SEC as described in "Materials and methods". Western SDS PAGE analysis of the tetraspanins CD63 and CD9; b quantification for both tetraspanins; ${ }^{*} n=7, p<0.05$.

\section{Synaptically released tau demonstrates seeding activity}

In order to test seeding potential of synaptic tau, we depolarized a series of three P-2 samples, starting again with relatively large P-2 sample sizes. Experiments used the FRET tau biosensor assay developed by Diamond and colleagues, which can detect seeding activity in samples before histopathological stains using flow cytometry analysis of HEK293 cells ${ }^{52}$. To eliminate smaller tau peptides that are free-floating, we concentrated each sample to about $20 \mu \mathrm{l}$ using Vivaspin centrifugal concentrators with a molecular weight cutoff of $100 \mathrm{kDa}$. Figure $3 \mathrm{~b}$ shows the seeding potential of $3 \mathrm{AD}$ cases compared to control; note that the AD1 and 2 cases used for Fig. $3 \mathrm{~b}$ are the same cases as AD1 (Table 1 case 3-13) and AD2 (Table 1 case 5-13) from Fig. 3a. Case AD1 showed much higher exosomal tau and tau oligomer levels compared to AD2, and demonstrated significantly higher seeding activity. There was not sufficient sample to include case AD4, which had the highest seeding activity, in the previous exosome immunoprecipitation experiment (Fig. 3a).

In order to compare the seeding potential of exosomal and freefloating tau, we initially attempted to concentrate the flowthrough from the pan exosome IP experiment above (Fig. 3a), but no seeding potential was detected despite multiple efforts with large samples. Therefore, we next purified exosomes from the release supernatants and separated fractions (F) by SEC. Synaptosomes of $A D$ cases were depolarized as described above, and the release supernatants collected. Exosomes were purified by ultracentrifugation and separated on SEC columns. Fractions 5-9 were collected and Western analysis showed the tetraspanin CD63 and tau in F6 and F7; the tau bands were very faint bands for tau monomer and a HMW tau oligomer at the top of the gel (Fig. 3c). A faint tau monomer band was sometimes observed in fractions other than F6 and F7; for example in fraction F5 (Fig. 3c). These bands most likely represent free-floating tau, since seeding activity was never observed in any fraction besides F6 and F7. In some experiments, CD63 and seeding activity but not tau bands were visible, consistent with loss of the very weak tau signal by dilution and separation with SEC. The size analysis (Fig. $3 \mathrm{~d}$ ) verifies a mean particle diameter with a mean of 144 and a mode of $106 \mathrm{~nm}$, consistent with exosomes. Tau seeding activity in HEK293 cells was also concentrated in fractions F6 and F7 (Fig. 3e). The localization of tau and exosomes to SEC fractions F6 and F7 was confirmed by a control experiment using commercial tau fibrils and standard human plasma exosomes; commercial tau fibrils (PFF) were run in alternating lanes with standard exosomes plus PFF (Fig. 3f). A comparison of released exosomes from APOE3 and $A P O E 4$ cases is shown in Fig. 4a; seeding activity did not differ by genotype (not shown), although Western analysis of CD63 from F6 indicates increased exosome release in APOE4 cases (Fig. 4b). The tetraspanin CD9 showed a similar trend that was not significant. Taken all together, these experiments demonstrate the colocalization of seeding activity, exosomes, and tau to fractions F6 and F7, demonstrating that the seeding activity of synaptically released tau is associated with exosomal but not free-floating tau.

\section{Tau seeding potential is reduced in pure tauopathy cortex compared to $A D$, but enhanced by amyloid beta}

We next asked how seeding activity of tauopathy cases without amyloid plaques compares to seeding activity in $A D$ cases with plaques and tangles, when tau concentrations are similar. For these experiments, cortical synaptosomes (P-2) were used as seeds, since, as noted above (Fig. 3b, e), the seeding activity of synaptosome release fractions is small and requires substantial amounts of starting material. Two aged cognitively normal parietal cortex samples were used as controls; to avoid confounds related to amyloid, five tauopathy cases were selected with no plaque pathology in parietal cortex, and Braak III-IV tau pathology (i.e., no neortical tau). Three AD cases with both tau and plaques in the parietal cortex were included. Soluble P-2 extracts were prepared, and both tau and $A \beta 42$ were measured by alphaLISA immunoassay; extracts were then applied to HEK293 biosensor cells, adjusting the volume so that each well contained $\sim 100 \mathrm{pg}$ tau for each sample. As expected, the control cases showed little seeding activity; Fig. 5a shows that the tau seeding activity was approximately fourfold higher in the two late $A D$ cases, with plaques and tangles in the cortex compared to the five tauopathy cases. Seeding activity was increased in both tauopathy and $A D$ cases compared to control (Fig. 5a; ANOVA $F(2,17=6.35$ ), $p=$ $0.0004)$. The $A \beta 42$ levels for each case are shown in Fig. $5 b$; even in the absence of plaques, there were variable low-to-moderate levels of $A \beta 42$ in one of the normal (NL1, Table 1 case 1-13,) and the tauopathy cases, consistent with a physiological role for $A \beta$ peptides in regulation of synaptic function ${ }^{53}$. Case AD6 (Table 1 case 7-11) showed fourfold less seeding activity than the other $A D$ cases; this $A D$ case had cognitive impairment but no dementia and intermediate pathology: Braak III, with no tangles but moderate $A \beta 42$ plaques in parietal cortex. The $A \beta 42$ level of the three $A D$ cases did not differ significantly, and all three were rated neuritic plaque level B-C by the neuropathologist; however, the seeding capacity of the two late stage cases, AD7 (Table 1 case 2311) and AD8 (Table 1 case 12-12), jumped fourfold (Fig. 5c). Even with the very low measured levels of $A \beta 42$ in the tauopathy cases with no plaques, seeding activity showed a significant correlation with the A 342 level $(r=0.79, p=0.007$; Fig. $5 c)$; but not with total $\operatorname{tau}(r=0.08$; not shown). The correlation with Braak \& Braak stage was also not significant ( $r=0.56$; not shown). Taken together, these results indicate that tau seeding activity is strongly associated with $A \beta 42$.

\section{DISCUSSION}

A large literature provides direct support for the trans-synaptic spread of tau pathology in $A D$, but virtually all studies use in vitro or animal models. In the present work, using human cryopreserved cortical tissue throughout, we developed a flow cytometry assay to quantify depolarization of human synaptosomes. Reduced fluorescence from the membrane dye FM2-10 verifies $\mathrm{KCl}$-induced depolarization, and the viability dye calcein AM 


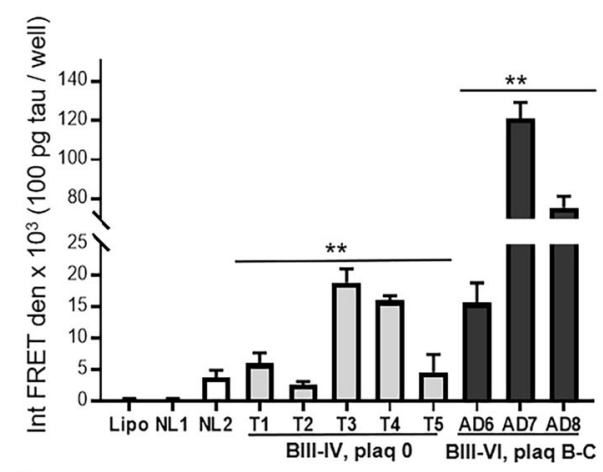

b
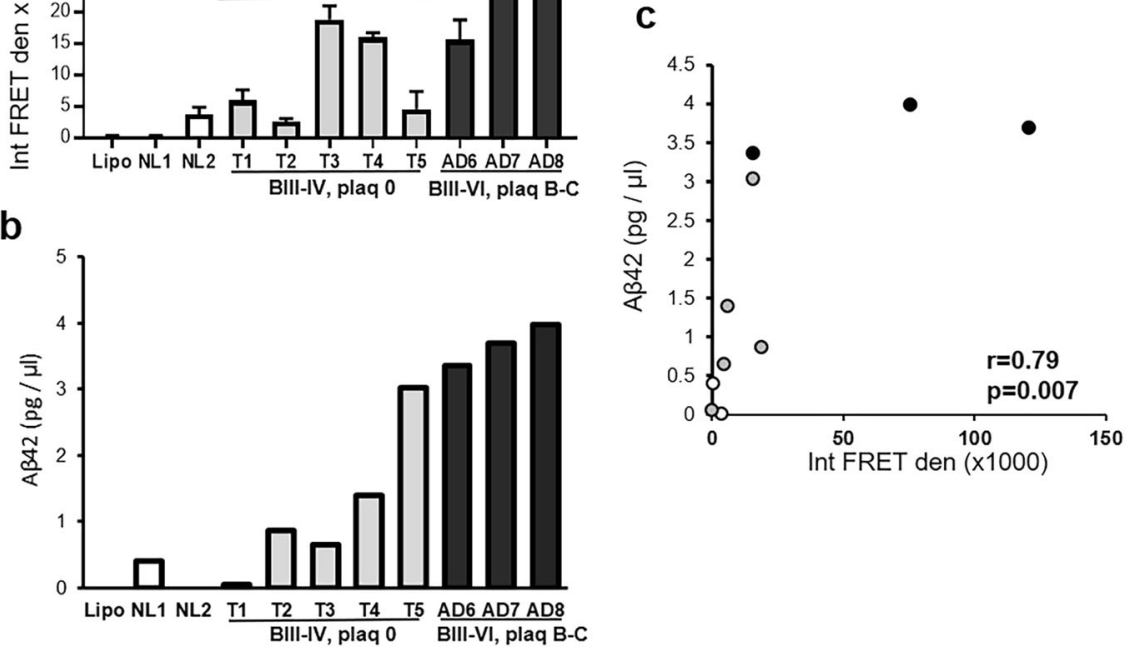

Fig. 5 Tauopathy samples demonstrate low seeding potential compared to AD. a Tau biosensor cells were seeded with aliquots of cortical P-2 from normal controls (NL1, NL2; white), tauopathy cases without plaques (Braak III-IV, plaque 0; T1-5; gray), and AD cases (Braak III-VI, plaque B,C; AD6-8; black), with lipofectamine control (Lipo). Integrated FRET density (Int FRET den). Seeding activity was increased in tauopathy and in AD compared to controls; ${ }^{* *} p<0.01$, one-way ANOVA. b Soluble A $\beta 42$ was measured in the P-2 from the same cases used in (a). c Correlation analysis of $A \beta 42$ level and integrated FRET density.

shows that depolarized synaptosomes remain intact. TEM images show exosome-sized particles in release supernatants, and markers of multiple EV-associated tetraspanin proteins are increased by depolarization. Antibodies indicate that some released tau is phosphorylated and oligomeric, and that most released tau is C-terminal-truncated. Immunoprecipitation with tetraspanin proteins (CD9, CD63, and CD81) definitively localized significant tau peptides and oligomers onto EVs. Importantly, when synaptic release supernatants were fractionated by size, the exosome marker CD63 and tau were observed in the only fractions to demonstrate seeding activity. This indicates that exosomal rather than free-floating tau is the basis for transynaptic spread of tau pathology. In tau seeding experiments with crude synaptosomes (P-2) from control, tauopathy, and AD cases, seeding activity was highly associated with the low levels of $A \beta 42$ detected in tauopathy cortex samples without plaques. However, in these cases, seeding was fourfold less than the maximum seeding activity measured when both plaques and tangles were present in $A D$ cortical samples, indicating that $A \beta$ makes a major contribution to seeding activity.

It is important to note that the FM2-10 assay shows robust depolarization, and that the degree of depolarization in fresh mouse synaptosomes was identical to the cryopreserved human synaptosomes. It is also clear that tau and exosome release occurs at rest in control buffer and the increase with $\mathrm{KCl}$ depolarization is fairly modest but significant. We attempted a more thorough characterization of released tau peptides, but it is important to emphasize the extremely low protein and tau levels in the in vitro release supernatants. For example, acetylated tau, caspase-cleaved tau, and additional p-tau epitopes (PHF1, PS396) were not detected (not shown). In the present results, release of fragments and various tau oligomers was observed, especially at about the size of trimers, and exosomal tau from one $A D$ cortical sample (Fig. 3a) showed a dense laddering and smearing pattern above and below the molecular weight of the intact protein at $\sim 55 \mathrm{kDa}$. This is similar to previous observations by us and others ${ }^{21,22,54}$, and is consistent with accumulation and extensive aggregation of multiple tau fragments rather than one or several key tau cleavage fragments.

Our finding that truncated tau is released would be expected based on our previous result that most synaptosomal tau is Cterminal-truncated ${ }^{50}$; this result is consistent with some observations but there is no consensus in the literature. For example, freefloating-truncated tau was released from human induced pluripotent stem cell (iPSC)-derived neurons, while full-length tau was detected in exosomes ${ }^{13}$; Wang and colleagues also found exosomal tau to be full length ${ }^{6}$. Most human tau secreted by Hela cells was C-terminal-truncated ${ }^{55}$, and several in vitro studies suggest that C-terminal-truncated tau and full-length tau are secreted by different mechanisms ${ }^{56,57}$, although another group, also using cultured neurons, found intracellular tau to be full length, while the majority of extracellular tau was truncated ${ }^{17}$.

Several papers note that only a small fraction of tau is exosomal, with the vast majority of extracellular tau free-floating ${ }^{6,13}$, and free-floating tau assemblies equal to or greater than trimers have been shown to seed aggregation ${ }^{18}$. In the present work, we were unable to seed with concentrated free-floating tau extracts despite multiple efforts. However, SEC separation of released exosomes clearly shows that CD63 and tau are associated with the two fractions that demonstrate seeding activity. The small monomeric tau signals seen occasionally in other fractions, probably representing free-floating tau, were not associated with seeding activity. Along this line, in some experiments, only very faint HMW tau oligomers were present in Fractions 6 and 7, and in some experiments, the tau signal was not visible in those fractions but CD63 and seeding activity were observed. Since others have shown seeding from extracellular tau, it may be that there is less free-floating tau and more exosomal tau from human cortical 
synapses. It is also possible that very low levels of HMW freefloating aggregates with high seeding potential ${ }^{19,20}$ were present below the assay threshold for seeding activity, an important point since the argument has been made that the HEK biosensor assay represents a non-physiologic form of aggregation ${ }^{58}$. More work is needed to address this issue, but our inability to show seeding activity of the flow-through containing free-floating tau, and the colocalization of released tau and exosomes in fractions with seeding activity is most consistent with the hypothesis that tau spread is exosomal in human AD.

With respect to phosphorylated tau, multiple p-tau epitopes and high levels of $p$-tau have long been observed in AD tissue and in multiple mouse models, and are detected in synaptosomes ${ }^{21,59,60}$, but there is general agreement in the literature that extracellular tau is relatively hypophosphorylated in multiple model systems. In the present experiments, the released p-tau signal was very faint and only a single $p$-tau epitope was detected. Similar to our results, in rTg4510 tau transgenic mice, Polanco and colleagues found exosomal tau to be weakly phosphorylated compared to the multiple relatively abundant disease-associated epitopes found in their mouse model ${ }^{12}$. Clear hypophosphorylation of exosomal compared to cytosolic tau was also observed by Wang et al. in N2A cells and primary culture ${ }^{6}$. A potential mechanism is suggested by a similar pattern observed in 3xTg-AD slice cultures and rat cortical neurons, where dephosphorylated tau was observed in membraneassociated fractions ${ }^{61,62}$. These authors suggest that a pool of releasable tau peptides at the membrane awaiting secretion is regulated by phosphorylation. Tau also can be dephosphorylated after release by tissue-nonspecific alkaline phosphatase ${ }^{63}$, but dephosphorylation seems unlikely to affect tau inside EVs. However, a thorough study with human postmortem samples showed that HEK biosensor seeding activity was highly variable between cases, and was associated with disease progression and with phosphorylation on Thr231 and Ser235 or Ser262 but not with other p-tau epitopes $^{64}$. As noted above, we did not detect multiple p-tau epitopes, but given the barely detectable tau in our release supernatants, it is entirely possible that relevant phosphorylated peptides were below the detection limit of our assays.

Our results suggest a higher level of exosome release in the presence of APOE4, which might be expected, since exosomes are suggested as a mechanism for export of pathologic proteins. This is in contrast to a previous observation by Peng and colleagues, who demonstrated reduced exosome content in human samples and a mouse model expressing human apoE. The samples used were also cortical samples, but no AD was present, and EVs were isolated using enzymatic dissociation and ultracentrifugation ${ }^{65}$. These authors hypothesized reduced exosome biogenesis in the setting of $A P O E 4$, possibly contributing to $A D$ risk. In contrast, the present study focused on $A D$ samples and considered only synaptically released exosomes, likely to be responsible for the different results. It is also entirely possible for exosomes to have dual or multifocal actions in $A D^{66}$.

Though the literature for exosomal tau is far more robust, a number of papers clearly show $A \beta$ as an exosome cargo responsible for pathological transfer of $A \beta$ via exosomes $24,59,67,68$, so we were surprised that the extracellular $A \beta$ signal in our $A D$ samples was extremely faint. We did not examine our IP-isolated exosomes from synaptosome release for $A \beta$ (Fig. 3a), because there was a vanishingly small yield, and exosomal $A \beta$ would not be expected, given the low level of total extracellular $A \beta$ in Fig. 2 . In one study, with a design similar to ours, Bennett and colleagues found that homogenates from $A D$ brain regions with and without amyloid plaques showed increased seeding potential by two- to threefold, using the tau biosensor assay developed by Diamond and also used by $u s^{69}$. Similar to our result in which $A \beta$ but not tau level correlated with seeding activity, these authors concluded that bioactivity rather than level of tau was increased by $A \beta$, and that soluble $A \beta$, not just plaque $A \beta$, was capable for enhancement ${ }^{69}$.

Along this line, in previous work we have shown that the level of synaptic $\mathrm{p}$-tau is markedly higher in individual terminals positive for $A \beta$; using samples from hippocampus and entorhinal cortex in early stage disease samples ( $\leq$ Braak IV), we demonstrated a highly significant correlation between early terminal levels of $p$-tau and $A \beta^{60}$. We concluded that $A \beta$ may directly drive tau phosophorylation early in the disease process, consistent with synaptic $p$-tau induction that is directly driven within the terminal by protein-protein contact between synaptic oligomeric $A \beta$ and $\operatorname{tau}^{60}$. Such a prion-like mechanism occuring within synapses finds strong support in a paper by Vasconcelos et al., which showed heterotypic seeding of tau fibrillization by aggregated $A \beta$ in a cellfree system ${ }^{70}$. As noted by these authors and by others ${ }^{22,69-72}$, prion-like seeding of tau by $A \beta$ explains a number of peculiarities of $A D$ compared to other tauopathies, including the initial appearance of $A \beta$ in isocortical regions ${ }^{73}$, and the clear $A \beta$ dependence of tau-associated cognitive loss ${ }^{14-76}$. Our tau seeding experiments from tauopathy and AD cases (Fig. 5) used P-2 samples; we have previously shown that the vast majority of synaptic $A \beta 42$ is oligomeric ${ }^{77}$. Interestingly, the $A \beta 42$ level in the three $A D$ cases is not significantly different, but the seeding activity for the two late stage cases AD2 and AD3 (Braak V, VI) jumps approximately fourfold from the level of activity of the Braak III stage AD1, a case with light pathology but without dementia (MMSE 26). This result highlights the strong correlation of tau pathology with cognition. However, the mechanism for the seeding activity jump is not clear although it illustrates the clear tau pathology acceleration by $A \beta$ seen by others ${ }^{22,69,70,78}$. It is possible that the $A \beta 42$ concentration has a sharp threshold for seeding activity; perhaps more likely, the sharp uptake is related to another $A \beta$ peptide that correlates with $A \beta 42$, perhaps oligomers or acetylated tau ${ }^{79}$.

The overall correlation between synaptic $A \beta 42$ level and biosensor activity is strong. Taken together with our earlier data showing more $p$-tau in $A \beta$-positive synapses ${ }^{60}$, and previous work by others ${ }^{70,80}$, the direct templating of misfolded tau by $A \beta$ oligomers within synapses may represent a plausible mechanism for the $A \beta$ boost in tau propagation. We cannot exclude that seeding activity is also driven by differences in tau conformers ${ }^{81,82}$, which is supported by a number of papers showing prion-like spread of misfolding between tau peptides ${ }^{83-87}$. On the other hand, a number of studies have clearly demonstrated that the presence of $A \beta$ accelerates tau aggregation, propagation, and cellular toxicity $22,69-71,78,88-90$ supporting a hypothesis that differences in terminal $A \beta$ amyloid load may be the primary reason for observed differences in tau seeding activity.

The extremely low levels of extracellular $A \beta$ in our results argues against the possibility that aggregated $A \beta$ directly templates misfolded tau within exosomes as a major mechanism, although $A \beta /$ tau direct contact might occur. Our data are most consistent with a model where misfolded aggregated tau, accelerated by terminal amyloid levels, would subsequently be loaded to exosomes and exported from the synapse, resulting in exosomal transfer of tau across synapses. More work is needed, but such a model is consistent with our data showing aggregated exosomal tau, the failure of free-floating tau to seed, and multiple reports by us and others of synaptic accumulations of misfolded, fibrillary $\operatorname{tau}^{22,24,69,91,92}$. The hypothesized model would support the consensus that amyloid is the upstream initiating pathology, and the observed failure of anti-amyloid therapies even when given early. This model, where $A \beta$ accelerates the aggregation of synaptic tau, may provide support for aggregation inhibitors ${ }^{93,94}$ and tau-targeted therapeutic approaches, including a reduction of secretion of tau-bearing exosomal subpopulations ${ }^{26,95}$. 


\section{DATA AVAILABILITY}

All the data generated or analyzed during this study are included in the manuscript.

\section{REFERENCES}

1. Braak H. \& Del Tredici K. Alzheimer's pathogenesis: is there neuron-to-neuron propagation? Acta Neuropathol. 121, 589-595 (2011).

2. Mohamed, N. V., Herrou, T., Plouffe, V., Piperno, N. \& Leclerc, N. Spreading of tau pathology in Alzheimer's disease by cell-to-cell transmission. Eur. J. Neurosci. 37, 1939-1948 (2013)

3. Kfoury, N., Holmes, B. B., Jiang, H., Holtzman, D. M. \& Diamond, M. I. Trans-cellular propagation of Tau aggregation by fibrillar species. J. Biol. Chem. 287, 19440-19451 (2012).

4. Medina, M. \& Avila, J. The role of extracellular Tau in the spreading of neurofibrillary pathology. Front. Cell Neurosci. 8, 113 (2014).

5. de Calignon, A. et al. Propagation of tau pathology in a model of early Alzheimer's disease. Neuron 73, 685-697 (2012).

6. Wang, Y. et al. The release and trans-synaptic transmission of Tau via exosomes. Mol. Neurodegener. 12, 5 (2017).

7. $\mathrm{Wu}, \mathrm{J}$. W. et al. Neuronal activity enhances tau propagation and tau pathology in vivo. Nat. Neurosci. 19, 1085-1092 (2016).

8. Sokolow, S. et al. Pre-synaptic C-terminal truncated tau is released from cortical synapses in Alzheimer's disease. J. Neurochem. 133, 368-379 (2015).

9. Kowal, J. et al. Proteomic comparison defines novel markers to characterize heterogeneous populations of extracellular vesicle subtypes. Proc. Natl Acad. Sci. U. S. A. 113, E968-977 (2016).

10. Stopschinski, B. E. \& Diamond, M. I. The prion model for progression and diversity of neurodegenerative diseases. Lancet Neurol. 16, 323-332 (2017).

11. Schneider, A. \& Simons, M. Exosomes: vesicular carriers for intercellular communication in neurodegenerative disorders. Cell Tissue Res. 352, 33-47 (2013).

12. Polanco, J. C., Scicluna, B. J., Hill, A. F. \& Gotz, J. Extracellular vesicles isolated from the brains of $\mathrm{rTg} 4510$ mice seed Tau protein aggregation in a thresholddependent manner. J. Biol. Chem. 291, 12445-12466 (2016).

13. Guix, F. X. et al. Detection of aggregation-competent Tau in neuron-derived extracellular vesicles. Int. J. Mol. Sci. 19, 663-686 (2018).

14. Dujardin, S. et al. Ectosomes: a new mechanism for non-exosomal secretion of tau protein. PLOS ONE 9, e100760 (2014).

15. Polanco, J. C., Li, C., Durisic, N., Sullivan, R. \& Gotz, J. Exosomes taken up by neurons hijack the endosomal pathway to spread to interconnected neurons. Acta Neuropathol. Commun. 6, 10 (2018).

16. Saman, S. et al. Exosome-associated tau is secreted in tauopathy models and is selectively phosphorylated in cerebrospinal fluid in early Alzheimer disease. J. Biol. Chem. 287, 3842-3849 (2012).

17. Kanmert, D. et al. C-terminally truncated forms of Tau, but not full-length Tau or its C-terminal fragments, are released from neurons independently of cell death. J. Neurosci. 35, 10851-10865 (2015).

18. Mirbaha, H., Holmes, B. B., Sanders, D. W., Bieschke, J. \& Diamond, M. I. Tau trimers are the minimal propagation unit spontaneously internalized to seed intracellular aggregation. J. Biol. Chem. 290, 14893-14903 (2015).

19. Takeda, S. et al. Neuronal uptake and propagation of a rare phosphorylated highmolecular-weight tau derived from Alzheimer's disease brain. Nat. Commun. 6, 8490 (2015).

20. Dujardin, S. et al. Tau molecular diversity contributes to clinical heterogeneity in Alzheimer's disease. Nat. Med. https://doi.org/10.1038/s41591-020-0938-9 (2020).

21. Henkins, K. M. et al. Extensive $p$-tau pathology and SDS-stable $p$-tau oligomers in Alzheimer's cortical synapses. Brain Pathol. https://doi.org/10.1111/j.17503639.2012.00598.x (2012).

22. $\mathrm{Wu}, \mathrm{H}$. Y. et al. beta-Amyloid induces pathology-related patterns of Tau hyperphosphorylation at synaptic terminals. J. Neuropathol. Exp. Neurol. 77, 814-826 (2018).

23. Lasagna-Reeves, C. A. et al. Tau oligomers impair memory and induce synaptic and mitochondrial dysfunction in wild-type mice. Mol. Neurodegener. 6, 39 (2011).

24. Sardar Sinha, M. et al. Alzheimer's disease pathology propagation by exosomes containing toxic amyloid-beta oligomers. Acta Neuropathol. 136, 41-56 (2018).

25. Dinkins, M. B., Dasgupta, S., Wang, G., Zhu, G. \& Bieberich, E. Exosome reduction in vivo is associated with lower amyloid plaque load in the 5XFAD mouse model of Alzheimer's disease. Neurobiol. Aging 35, 1792-1800 (2014).

26. Bilousova, T. et al. Suppression of tau propagation using an inhibitor that targets the DK-switch of nSMase2. Biochem. Biophys. Res. Commun. 499, 751-757 (2018).

27. Fiandaca, M. S. et al. Identification of preclinical Alzheimer's disease by a profile of pathogenic proteins in neurally derived blood exosomes: a case-control study. Alzheimers Dement. 11, 600-607.e1 (2015).

28. Herrmann, A. \& Spires-Jones, T. Clearing the way for tau immunotherapy in Alzheimer's disease. J. Neurochem. 132, 1-4 (2015).
29. Pooler, A. M., Noble, W. \& Hanger, D. P. A role for tau at the synapse in Alzheimer's disease pathogenesis. Neuropharmacology 76(Pt A), 1-8 (2014).

30. Castillo-Carranza, D. L. et al. Tau immunotherapy modulates both pathological tau and upstream amyloid pathology in an Alzheimer's disease mouse model. J. Neurosci. 35, 4857-4868 (2015).

31. Dodd, P. R. et al. Optimization of freezing, storage, and thawing conditions for the preparation of metabolically active synaptosomes from frozen rat and human brain. Neurochem. Pathol. 4, 177-198 (1986).

32. Gylys, K. H., Fein, J. A., Tan, A. M. \& Cole, G. M. Apolipoprotein E enhances uptake of soluble but not aggregated amyloid-beta protein into synaptic terminals. J. Neurochem. 84, 1442-1451 (2003).

33. Baldwin, M. L., Rostas, J. A. \& Sim, A. T. Two modes of exocytosis from synaptosomes are differentially regulated by protein phosphatase types $2 \mathrm{~A}$ and $2 \mathrm{~B}$. J. Neurochem. 85, 1190-1199 (2003).

34. Fein, J. A. et al. Co-localization of amyloid beta and tau pathology in Alzheimer's disease synaptosomes. Am. J. Pathol. 172, 1683-1692 (2008).

35. Sokolow, $\mathrm{S}$. et al. Isolation of synaptic terminals from Alzheimer's disease cortex. Cytometry A 81, 248-254 (2012).

36. Vella, L. J. et al. A rigorous method to enrich for exosomes from brain tissue. J. Extracell. Vesicles 6, 1348885 (2017)

37. Furman, J. L. et al. Widespread tau seeding activity at early Braak stages. Acta Neuropathol. 133, 91-100 (2017).

38. Dunkley, P. R., Jarvie, P. E. \& Robinson, P. J. A rapid Percoll gradient procedure for preparation of synaptosomes. Nat. Protoc. 3, 1718-1728 (2008).

39. Begley, J. G. et al. Cryopreservation of rat cortical synaptosomes and analysis of glucose and glutamate transporter activities, and mitochondrial function. Brain Res. Brain Res. Protoc. 3, 76-82 (1998).

40. Kuo, S. W. \& Dodd, P. R. Electrically evoked synaptosomal amino acid transmitter release in human brain in alcohol misuse. Neurosignals 19, 117-127 (2011).

41. Battista, N., Bari, M., Finazzi-Agro, A. \& Maccarrone, M. Anandamide uptake by synaptosomes from human, mouse and rat brain: inhibition by glutamine and glutamate. Lipids Health Dis. 1, 1 (2002).

42. Prieto, G. A. et al. Pharmacological rescue of long-term potentiation in Alzheimer diseased synapses. J. Neurosci. 37, 1197-1212 (2017).

43. Corera, A. T., Doucet, G. \& Fon, E. A. Long-term potentiation in isolated dendritic spines. PLOS ONE 4, e6021 (2009).

44. Betz, W. J., Mao, F. \& Smith, C. B. Imaging exocytosis and endocytosis. Curr. Opin. Neurobiol. 6, 365-371 (1996).

45. Clayton, E. L. \& Cousin, M. A. The molecular physiology of activity-dependent bulk endocytosis of synaptic vesicles. J. Neurochem. 111, 901-914 (2009).

46. Gylys, K. H., Fein, J. A. \& Cole, G. M. Quantitative characterization of crude synaptosomal fraction (P-2) components by flow cytometry. J. Neurosci. Res. 61, 186-192 (2000).

47. Andreu, Z. \& Yanez-Mo, M. Tetraspanins in extracellular vesicle formation and function. Front. Immunol. 5, 442 (2014).

48. Rajendran, L. et al. Alzheimer's disease beta-amyloid peptides are released in association with exosomes. Proc. Natl Acad. Sci. U. S. A. 103, 11172-11177 (2006).

49. Bilousova, T. et al. Apolipoprotein E/amyloid-beta complex accumulates in Alzheimer disease cortical synapses via Apolipoprotein E receptors and is enhanced by APOE4. Am. J. Pathol. 189, 1621-1636 (2019).

50. Sokolow S. et al. Presynaptic C-terminal truncated tau is released from cortical synapses in Alzheimer's disease. J. Neurochem. https://doi.org/10.1111/jnc.12991 (2014).

51. Baietti, M. F. et al. Syndecan-syntenin-ALIX regulates the biogenesis of exosomes. Nat. Cell Biol. 14, 677-685 (2012).

52. Holmes, C. et al. Long-term effects of Abeta42 immunisation in Alzheimer's disease: follow-up of a randomised, placebo-controlled phase I trial. Lancet $\mathbf{3 7 2}$, 216-223 (2008).

53. Brothers, H. M., Gosztyla, M. L. \& Robinson, S. R. The physiological roles of amyloid-beta peptide hint at new ways to treat Alzheimer's disease. Front. Aging Neurosci. 10, 118 (2018)

54. Maeda, S. et al. Increased levels of granular tau oligomers: an early sign of brain aging and Alzheimer's disease. Neurosci. Res. 54, 197-201 (2006).

55. Plouffe, V. et al. Hyperphosphorylation and cleavage at D421 enhance tau secretion. PLOS ONE 7, e36873 (2012).

56. Kim, W. et al. Interneuronal transfer of human tau between Lamprey central neurons in situ. J. Alzheimers Dis. 19, 647-664 (2010).

57. Perez, M., Cuadros, R., Hernandez, F. \& Avila, J. Secretion of full-length tau or tau fragments in a cell culture model. Neurosci. Lett. 634, 63-69 (2016).

58. Kaniyappan, S. et al. FRET-based Tau seeding assay does not represent prion-like templated assembly of Tau filaments. Mol. Neurodegener. 15, 39 (2020).

59. Neddens, J. et al. Phosphorylation of different tau sites during progression of Alzheimer's disease. Acta Neuropathol. Commun. 6, 52 (2018). 
60. Bilousova, T. et al. Synaptic amyloid-beta oligomers precede p-Tau and differentiate high pathology control cases. Am. J. Pathol. 186, 185-198 (2016).

61. Pooler, A. M. et al. Dynamic association of tau with neuronal membranes is regulated by phosphorylation. Neurobiol. Aging 33, 431.e27-438 (2012).

62. Croft, C. L. et al. Membrane association and release of wild-type and pathological tau from organotypic brain slice cultures. Cell Death Dis. 8, e2671 (2017).

63. Diaz-Hernandez, M. et al. Tissue-nonspecific alkaline phosphatase promotes the neurotoxicity effect of extracellular tau. J. Biol. Chem. 285, 32539-32548 (2010).

64. Dujardin, S. et al. Tau molecular diversity contributes to clinical heterogeneity in Alzheimer's disease. Nat. Med. 26, 1256-1263 (2020).

65. Peng, K. Y. et al. Apolipoprotein E4 genotype compromises brain exosome production. Brain 142, 163-175 (2019).

66. Soares Martins, T. et al. Diagnostic and therapeutic potential of exosomes in Alzheimer's disease. J. Neurochem. 156, 162-181 (2021).

67. Lim, C. Z. J. et al. Subtyping of circulating exosome-bound amyloid beta reflects brain plaque deposition. Nat. Commun. 10, 1144 (2019).

68. Micci, M. A. et al. Hippocampal stem cells promotes synaptic resistance to the dysfunctional impact of amyloid beta oligomers via secreted exosomes. Mol. Neurodegener. 14, 25 (2019).

69. Bennett, R. E. et al. Enhanced Tau aggregation in the presence of amyloid beta. Am. J. Pathol. 187, 1601-1612 (2017).

70. Vasconcelos, B. et al. Heterotypic seeding of Tau fibrillization by pre-aggregated Abeta provides potent seeds for prion-like seeding and propagation of Taupathology in vivo. Acta Neuropathol. 131, 549-569 (2016).

71. Gotz, J., Chen, F., van Dorpe, J. \& Nitsch, R. M. Formation of neurofibrillary tangles in P301I tau transgenic mice induced by Abeta 42 fibrils. Science 293, 1491-1495 (2001).

72. Guerrero-Munoz, M. J., Gerson, J. \& Castillo-Carranza, D. L. Tau oligomers: the toxic player at synapses in Alzheimer's disease. Front. Cell. Neurosci. 9, 464 (2015).

73. Thal, D. R., Rub, U., Orantes, M. \& Braak, H. Phases of A beta-deposition in the human brain and its relevance for the development of AD. Neurology 58, 1791-1800 (2002).

74. Jack, C. R. Jr. et al. Tracking pathophysiological processes in Alzheimer's disease: an updated hypothetical model of dynamic biomarkers. Lancet Neurol. 12, 207-216 (2013)

75. Mann, D. M. \& Hardy, J. Amyloid or tau: the chicken or the egg? Acta Neuropathol. 126, 609-613 (2013).

76. Jack, C. R. Jr. et al. Hypothetical model of dynamic biomarkers of the Alzheimer's pathological cascade. Lancet Neurol. 9, 119-128 (2010).

77. Sokolow, S. et al. AD synapses contain abundant Abeta monomer and multiple soluble oligomers, including a 56-kDa assembly. Neurobiol. Aging 33, 1545-1555 (2012).

78. Pooler, A. M. et al. Amyloid accelerates tau propagation and toxicity in a model of early Alzheimer's disease. Acta Neuropathol. Commun. 3, 14 (2015).

79. Tracy T. E. \& Gan L. Acetylated tau in Alzheimer's disease: an instigator of synaptic dysfunction underlying memory loss: increased levels of acetylated tau blocks the postsynaptic signaling required for plasticity and promotes memory deficits associated with tauopathy. Bioessays 39, 1-9 (2017).

80. Guerrero-Munoz, M. J. et al. Amyloid-beta oligomers as a template for secondary amyloidosis in Alzheimer's disease. Neurobiol. Dis. 71, 14-23 (2014).

81. Zhang, W. et al. Novel tau filament fold in corticobasal degeneration. Nature $\mathbf{5 8 0}$ 283-287 (2020).

82. Arakhamia, T. et al. Posttranslational modifications mediate the structural diversity of tauopathy strains. Cell 180, 633-644.e12 (2020).

83. Sanders, D. W. et al. Distinct tau prion strains propagate in cells and mice and define different tauopathies. Neuron 82, 1271-1288 (2014).

84. Woerman, A. L. et al. Tau prions from Alzheimer's disease and chronic traumatic encephalopathy patients propagate in cultured cells. Proc. Natl Acad. Sci. U. S. A. 113, E8187-E8196 (2016).

85. Clavaguera, F. et al. Transmission and spreading of tauopathy in transgenic mouse brain. Nat Cell Biol 11, 909-913 (2009).

86. Frost, B., Jacks, R. L. \& Diamond, M. I. Propagation of tau misfolding from the outside to the inside of a cell. J Biol. Chem. 284, 12845-12852 (2009).

87. Woerman, A. L. et al. Kinetics of human mutant Tau prion formation in the brains of 2 transgenic mouse lines. JAMA Neurol. 74, 1464-1472 (2017).

88. Chabrier, M. A. et al. Soluble abeta promotes wild-type tau pathology in vivo. J. Neurosci. 32, 17345-17350 (2012).

89. Ittner, L. M. et al. Dendritic function of tau mediates amyloid-beta toxicity in Alzheimer's disease mouse models. Cell 142, 387-397 (2010).
90. De Felice, F. G. et al. Alzheimer's disease-type neuronal tau hyperphosphorylation induced by A beta oligomers. Neurobiol. Aging 29, 1334-1347 (2008).

91. Henkins, K. M. et al. Extensive p-tau pathology and SDS-stable p-tau oligomers in Alzheimer's cortical synapses. Brain Pathol. 22, 826-833 (2012).

92. Maeda, S. et al. Granular tau oligomers as intermediates of tau filaments. Biochemistry 46, 3856-3861 (2007).

93. Malik, R. et al. Using molecular Tweezers to remodel abnormal protein selfassembly and inhibit the toxicity of amyloidogenic proteins. Methods Mol. Biol. 1777, 369-386 (2018).

94. Lo Cascio, F. et al. Toxic Tau oligomers modulated by novel curcumin derivatives. Sci. Rep. 9, 19011 (2019).

95. Asai, H. et al. Depletion of microglia and inhibition of exosome synthesis halt tau propagation. Nat. Neurosci. 18, 1584-1593 (2015).

\section{AUTHOR CONTRIBUTIONS}

Participated in research design: E.M., T.B., V.J., and K.H.G. Conducted experiments: E.M., T.B., M.M., D.F., C.E., K.N.M., and J.J.C. Autopsy sample collection and analysis: W.W.P., H.V.V., C.A.M., M.C., C.K., R.B., and C.C. Performed data analysis: E.M., T.B., M.M., and K.H G. Wrote or contributed to the writing of the manuscript: T.B., M.M., and K.H.G.

\section{FUNDING}

This work was supported by NIH AG063767 to T.B. and K.H.G., AG051946 to K.H.G., NIH AG18879 to C.A.M., and NIH AG051386 to V.J. The ARCs Foundation provided support for M.M. Tissue was obtained from the AD Research Center Neuropathology Cores of USC (NIA P50 AG05142), UCLA (NIA P50 AG16570), and UC Irvine (NIA P30AG016573 and P30AG066519). Flow cytometry was performed in the UCLA Jonsson Comprehensive Cancer Center (JCCC) and Center for AIDS Research Flow Cytometry Core Facility supported by NIH CA16042 and Al28697, and by the JCCC, the UCLA AIDS Institute, the David Geffen School of Medicine and the Chancellor's Office at UCLA. Diagnosis, characterization, and follow-up of $90+$ Study subjects were supported by NIA R01AG021055. We thank Mari Perez-Rosendahl and Ronald C. Kim for UCI Neuropathology reports.

\section{COMPETING INTERESTS}

The authors declare no competing interests.

\section{ETHICS APPROVAL AND CONSENT TO PARTICIPATE}

All authors read and approved the final manuscript.

\section{ADDITIONAL INFORMATION}

Correspondence and requests for materials should be addressed to K.H.G.

Reprints and permission information is available at http://www.nature.com/ reprints

Publisher's note Springer Nature remains neutral with regard to jurisdictional claims in published maps and institutional affiliations.

Open Access This article is licensed under a Creative Commons Attribution 4.0 International License, which permits use, sharing, adaptation, distribution and reproduction in any medium or format, as long as you give appropriate credit to the original author(s) and the source, provide a link to the Creative Commons license, and indicate if changes were made. The images or other third party material in this article are included in the article's Creative Commons license, unless indicated otherwise in a credit line to the material. If material is not included in the article's Creative Commons license and your intended use is not permitted by statutory regulation or exceeds the permitted use, you will need to obtain permission directly from the copyright holder. To view a copy of this license, visit http://creativecommons org/licenses/by/4.0/.

(c) The Author(s) 2021 